\title{
Sequential solution of the sideways heat equation by windowing of the data
}

\author{
Fredrik Berntsson
}

Linköping University Post Print

N.B.: When citing this work, cite the original article.

Original Publication:

Fredrik Berntsson, Sequential solution of the sideways heat equation by windowing of the data, 2003, Inverse Problems in Engineering, (11), 2, 91-103.

http://dx.doi.org/10.1080/1068276021000048564

Copyright: Taylor \& Francis

http://www.tandf.co.uk/journals/default.asp

Postprint available at: Linköping University Electronic Press

http://urn.kb.se/resolve?urn=urn:nbn:se:liu:diva-46683 


\title{
Sequential Solution of the Sideways Heat Equation by Windowing of the Data
}

\author{
Fredrik Berntsson*
}

\begin{abstract}
The sideways heat equation is a one dimensional model of a problem, where one wants to determine the temperature on the surface of a body using interior measurements. More precisely, we consider a heat conduction problem, where temperature and heat-flux data are available along the line $x=1$ and the solution is sought in the interval $0 \leq x<1$.

The problem is ill-posed in the sense that the solution does not depend continuously on the data. Stability can be restored by replacing the time derivative in the heat equation with a bounded spectral approximation. The cut off level in the spectral approximation acts as a regularization parameter, that controls the degree of smoothness in the solution.

In certain applications one wants to solve the sideways heat equation in real time, i.e. to constantly update the solution as new measurements are recorded. For this case sequential solution methods are required.
\end{abstract}

Key words. Ill-posed, Sideways Heat Equation, Sequential method

\section{Introduction}

In several applications one wishes to determine the temperature history on the surface of a body. However, often the surface itself is inaccessible for measurements. It may also be the case that locating a measurement device on the surface would disturb the measurements so that an incorrect temperature would be recorded. In such cases one might attempt to use measurements, taken in the interior of the body, and compute the desired surface temperature, by solving the heat equation numerically.

The sideways heat equation is a model of this situation: Find the temperature distribution $T(x, t)$ that satisfies,

$$
\begin{array}{ll}
\kappa \frac{\partial^{2} T}{\partial x^{2}}=\frac{\partial T}{\partial t}, & x \in[0,1], t \in \mathbb{R}, \\
T(1, t)=g(t), & t \in \mathbb{R}, \\
\frac{\partial T}{\partial x}(1, t)=h(t), & t \in \mathbb{R},
\end{array}
$$

where $\kappa>0$ is the thermal diffusivity.

*Department of Mathematics, Linköping University, S-581 83 Linköping, Sweden. e-mail: frber@math.liu.se 


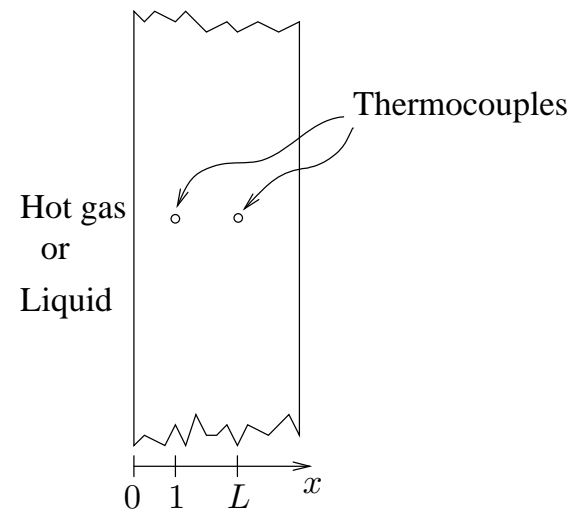

Figure 1: Temperature measurements in the interior of a wall.

Of course, since $g$ and $h$ have to be measured, we would actually have data functions $g_{m}, h_{m}$ in $L^{2}(\mathbb{R})$ satisfying,

$$
\left\|g_{m}-g\right\|<\varepsilon_{1}, \quad \text { and, } \quad\left\|h_{m}-h\right\|<\varepsilon_{2},
$$

where $\varepsilon_{1}$ and $\varepsilon_{2}$ represent bounds on the measurement errors.

In practice it is difficult to accurately measure the heat-flux $\partial_{x} T$ in the interior of the body. However, often it is the case that the heat equation is actually valid in a larger interval $[0, L]$, cf. Figure 1 . Thus, if the temperature history $T(L, t)$ is also recorded, then the heat flux $\partial_{x} T(1, t)$ can be computed by solving a well-posed boundary value problem, for the heat equation, in the interval $x \in[1, L]$.

Consider a practical situation where the temperature and heat-flux data are continuously recorded at a location inside a body. Clearly, at any given moment, measurements will only be available for a finite period of time. Therefore, it is only reasonable to attempt to solve (1) for the time period where data are available. Furthermore, when new measurements are recorded we wish to update our existing solution, by making use of the newly acquired data. For this case sequential solution methods are required [1, 11]. The most popular methods for solving the sideways heat equation cannot be used directly since they require that the temperature and heat-flux data, for the whole time domain, are collected before the solution can be computed. In particular this is true for the methods based on replacing the time derivative by a spectral or wavelet approximations $[2,6,15]$. Methods based on mollification $[9,13,14,18]$ can either be considered as full-domain methods, or as sequential in time, depending on their implementation. In this paper a simple way of transforming full-domain solution methods into sequential procedures, that can be used for constantly monitoring the surface temperature, is proposed.

Although, in this paper, we mostly consider the heat equation in its simplest form, $\kappa \partial_{x}^{2} T=\partial_{t} T,{ }^{1}$ more general equations, e.g. equations with non-constant coefficients,

$$
\frac{\partial}{\partial x}\left(\kappa(x) \frac{\partial T}{\partial x}\right)=\frac{\partial T}{\partial t}
$$

\footnotetext{
${ }^{1}$ The abbreviation $\partial_{x}=\frac{\partial}{\partial x}$ is used in this paper.
} 
or non-linear equations,

$$
\frac{\partial}{\partial x}\left(\kappa(T) \frac{\partial T}{\partial x}\right)=\frac{\partial T}{\partial t},
$$

frequently appear in applications. The sequential procedure that is proposed in this paper is not applicable for the latter case.

The organization of this paper is as follows. In Section 2 we analyze the sideways heat equation, using the Fourier transform, and demonstrate that it is ill-posed. In Section 3 it is shown that the sideways heat equation can be solved sequentially in time, by taking advantage of the fact the heat equation is linear. The implementation of numerical methods for solving the sideways heat equation is briefly discussed in Section 4. A numerical example, intended to illustrate the properties of the proposed method, is given in Section 5. Finally, in Section 6, there is a short discussion about the advantages and limitations of the proposed method.

\section{Ill-posedness and stabilization}

The sideways heat equation is ill-posed in the sense that the solution, if it exists, does not depend continuously on the data. Here we give an analysis, which demonstrates the fundamental reason for this ill-posedness, and also suggests a natural way to numerically stabilize the problem. For simplicity we initially assume that $\kappa=1$.

Let,

$$
\widehat{T}(x, \xi)=\frac{1}{\sqrt{2 \pi}} \int_{-\infty}^{\infty} \mathrm{e}^{-i \xi t} T(x, t) d t,
$$

denote the Fourier transform of $T$. Then, in frequency space, the solution of (1) can easily be verified to be,

$$
\widehat{T}=\frac{1}{2}\left(\mathrm{e}^{\sqrt{i \xi}(1-x)}\left(\widehat{g}-\frac{\widehat{h}}{\sqrt{i \xi}}\right)+\mathrm{e}^{-\sqrt{i \xi}(1-x)}\left(\widehat{g}+\frac{\widehat{h}}{\sqrt{i \xi}}\right)\right) .
$$

The principal value of $\sqrt{i \xi}$ has a positive real part. Thus, for $0<x<1$, we have,

$$
\left|\mathrm{e}^{\sqrt{i \xi}(1-x)}\right|=\mathrm{e}^{\sqrt{|\xi| / 2}(1-x)} \rightarrow \infty, \quad \text { as } \quad|\xi| \rightarrow \infty .
$$

Hence, small errors in the high frequency components of the data $[\widehat{g}, \widehat{h}]$ will be magnified, and can completely destroy the solution.

A natural way to stabilize the problem is to introduce a "cut off" frequency $\xi_{c}$, and define a regularized solution,

$$
T_{c}(x, t)=\frac{1}{\sqrt{2 \pi}} \int_{-\xi_{c}}^{\xi_{c}} \mathrm{e}^{i \xi t} \widehat{T}(x, \xi) d \xi
$$

Similarly, we denote the regularized solution with measured data $\left[g_{m}, h_{m}\right]$ by $T_{c}^{\delta}(x, t)$. The difference between the solutions $T$ and $T_{c}^{\delta}$ can be divided into two parts,

$$
\left\|T(x, \cdot)-T_{c}^{\delta}(x, \cdot)\right\| \leq R_{T}+R_{X}
$$


where,

$$
R_{T}^{2}=\int_{|\xi|>\xi_{c}}|\widehat{T}(x, \xi)|^{2} d \xi, \text { and, } R_{X}^{2}=\int_{-\xi_{c}}^{\xi_{c}}\left|\widehat{T}_{c}-\widehat{T}_{c}^{\delta}\right|^{2} d \xi .
$$

These two terms behave differently. The truncation error $R_{T}$ measures the effect of the introduced cut off frequency, and by increasing $\xi_{c}$ we reduce $R_{T}$. The second term $R_{X}$, the propagated data error, is a consequence of the inexact data, and grows when $\xi_{c}$ is increased.

There is an optimal value for $\xi_{c}$, but unfortunately it cannot be computed explicitly since it depends on unavailable information, e.g. the smoothness of the exact data $[g, h]$. However it is possible to give a parameter choice rule which, given some $a$ priori information about the solution, leads to a convergent scheme [7, Sec. 3], that is

$$
\left\|T(x, \cdot)-T_{c}^{\delta}(x, \cdot)\right\| \rightarrow 0, \quad \text { if } \quad\left\|g_{m}-g\right\|,\left\|h_{m}-h\right\| \rightarrow 0 .
$$

Such a scheme is referred to as a Regularization method for solving the ill-posed problem [7].

The appropriate choice for $\xi_{c}$, based on a priori bounds on the solution and the noise level, was discussed in [2], see also [6]. More precisely, suppose that the heat equation is valid for $0 \leq x \leq L$, where $L \geq 3$, and that the bounds $\|T(x, \cdot)\| \leq M$ and $\left\|g-g_{m}\right\|+\left\|h-h_{m}\right\| \leq \delta$ holds. Then, if $\xi_{c}=2 \kappa(\log (M / \delta))^{2}$, and that with this choice $\xi_{c}>2 \kappa$, the error estimate,

$$
\left\|T(x, \cdot)-T_{c}^{\delta}(x, \cdot)\right\| \leq 2.5 M^{1-x} \delta^{x}, \quad 0 \leq x \leq 1,
$$

holds. The above error bound is of Hölder type, and is similar to those obtained by other authors $[4,6,12,17]$, for slight variations of this problem.

We conclude this section by giving an alternate analysis, which, more clearly, displays the fundamental reason why the problem is ill-posed, and also suggests how the problem should be solved numerically. Furthermore, the argument that is used here remain valid also for equations with non-constant coefficients.

Let $V(x, t)=\kappa \partial_{x} T(x, t)$, and rewrite (1) as an initial value problem for a system of first order equations,

$$
\frac{\partial}{\partial x}\left(\begin{array}{c}
T \\
V
\end{array}\right)=\left(\begin{array}{cc}
0 & \kappa^{-1} \\
\frac{\partial}{\partial t} & 0
\end{array}\right)\left(\begin{array}{c}
T \\
V
\end{array}\right), \quad 0 \leq x \leq 1
$$

with initial values,

$$
T(1, t)=g(t), \text { and }, V(1, t)=\kappa h(t), \quad t \in \mathbb{R} .
$$

Formally, the solution can be written,

$$
\left(\begin{array}{l}
T \\
V
\end{array}\right)=\mathrm{e}^{\mathcal{B}(x-1)}\left(\begin{array}{c}
\widehat{g} \\
\kappa \widehat{h}
\end{array}\right), \quad \mathcal{B}=\left(\begin{array}{cc}
0 & \kappa^{-1} \\
\frac{\partial}{\partial t} & 0
\end{array}\right),
$$

where the exponential function of the matrix $\mathcal{B}$ is defined in terms of its spectral decomposition. The spectrum of the operator $\mathcal{B}$ is unbounded in the right-half plane, and 


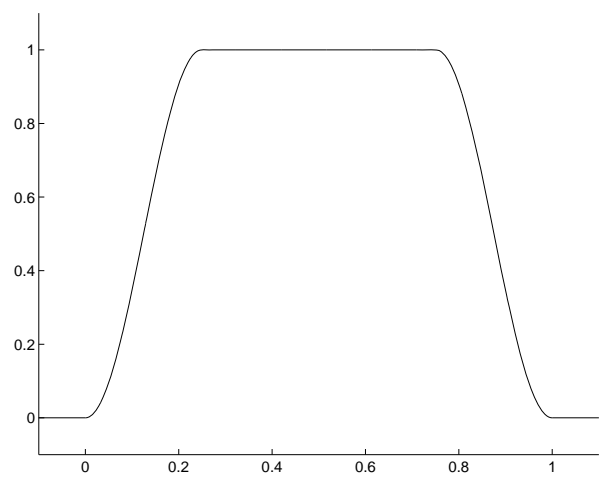

Figure 2: The function $w(t)$ that is used for the windowing procedure. Note that it is continuously differentiable.

therefore also the operator $\exp (\mathcal{B}(1-x))$ is unbounded. Hence the initial value problem (5) is ill-posed, and as a consequence the solution does not depend continuously on the data $[g, h]$.

The fundamental reason for this instability is that the operator $\partial / \partial t$ is unbounded. Thus if the time derivative is replaced by a bounded operator, then the resulting initial value problem is well-posed. Note, that computing the regularized solution (4) is, essentially, equivalent to solving the initial value problem (5), but where the derivative $\partial / \partial t$, i.e. the multiplication operator $i \xi$, is replaced by the bounded operator $i \xi \chi_{\left[-\xi_{c}, \chi_{c}\right]}(\xi) .^{2}$

\section{Continuous data monitoring}

In practice measurements will only be available for a finite period of time. In the remainder of this section $\mathcal{I}_{m}$ denotes the measurement interval, i.e. both $g_{m}$ and $h_{m}$ are known only for $t \in \mathcal{I}_{m}$. Here we will discuss an algorithm for computing the temperature history $T(0, t)$, for most of the interval $\mathcal{I}_{m}$. The procedure makes explicit use of the fact the the differential equation is linear. Therefore it can also be used for the case $\kappa=\kappa(x)$, but not if $\kappa=\kappa(T)$.

Let $w(t)$ be a compactly supported window function, with width $\ell$, such that,

$$
\sum_{k \in \mathbb{Z}} w(t-k \ell)=1
$$

An example of a function, which can be used as a window, is the following,

$$
w(t)= \begin{cases}\sin ^{2}(2 \pi t), & 0 \leq t<\frac{1}{4}, \\ 1, & \frac{1}{4} \leq t<\frac{3}{4}, \\ \cos ^{2}\left(2 \pi\left(t-\frac{3}{4}\right)\right), & \frac{3}{4} \leq t<1, \\ 0, & \text { otherwise. }\end{cases}
$$

\footnotetext{
${ }^{2} \chi_{[a, b]}$ denotes the characteristic function of the interval $[a, b]$.
} 
The width of this window is $\ell=3 / 4$. The basic idea is of course the trigonometric identity, $\sin ^{2}(t)+\cos ^{2}(t)=1$. The function $w(t)$ is illustrated in Figure 2. The initial data can be represented as follows,

$$
g(t)=\sum_{k \in \mathbb{Z}} g_{k}(t), \quad g_{k}(t)=w(t-k \ell) g(t) .
$$

The heat-flux data $h_{m}$ can be represented in a similar way. Denote by $T_{k}(x, t)$, for $k \in \mathbb{Z}$, the solution to the subproblem,

$$
\begin{array}{ll}
\kappa \frac{\partial^{2} T_{k}}{\partial x^{2}}=\frac{\partial T_{k}}{\partial t}, & x \in[0,1], t \in \mathbb{R}, \\
T_{k}(1, t)=g_{k}(t), & t \in \mathbb{R}, \\
\frac{\partial T_{k}}{\partial x}(1, t)=h_{k}(t), & t \in \mathbb{R} .
\end{array}
$$

Then, since the differential equation is linear, the solution to the original problem can be written as,

$$
T(x, t)=\sum_{k \in \mathbb{Z}} T_{k}(x, t) .
$$

Note that, since parts of the data functions $[g, h]$ are unknown, all terms in the series (10) cannot be computed. The data $\left[g_{k}, h_{k}\right]$, for a particular sub problem, are known completely only if the support of the shifted window $w(t-k \ell)$ is contained in $\mathcal{I}_{m}$.

The procedure described in this section can be summarized as follows.

Algorithm 1 Calculate $f(t)=T(0, t)$, for $t>0$, by

$$
\begin{aligned}
& f^{(0)}(t)=0 \\
& \text { for } k=0,1,2, \ldots \\
& \qquad \begin{array}{l}
g_{k}(t)=w(t-k \ell) g(t) \\
h_{k}(t)=w(t-k \ell) h(t) \\
\quad \text { compute } T_{k}(0, t) \quad \text { (by solving (9)) } \\
\quad f^{(k+1)}(t)=f^{(k)}(t)+T_{k}(0, t)
\end{array} \\
& \text { end }
\end{aligned}
$$

The desired solution is then $f(t)=\lim _{k \rightarrow \infty} f^{(k)}(t)$.

Note that, if the support of the window $w(t)$ is the interval $0<t<1$, then the data functions for the $k$ th subproblem are non-zero in the interval $k \ell<t<1+k \ell$. Thus, if the computations are to take place in real time, then the $k$ th step of the above algorithm must to be completed before $t=1+(k+1) \ell$. This is usually not very restrictive.

The technique described in this section works quite well, and is based on a simple observation. For illustrative purposes we consider a numerical example, where the heat equation, $\partial_{x}^{2} T=\partial_{t} T$, is valid for $0<x<5$, and the temperature is kept at $0{ }^{\circ} C$ at the boundary $x=5$. In Figure 3 we display the response, at $x=1$, if the surface is subject to a thermal impulse, corresponding to $T(0, t)=\delta(t)$, where $\delta$ denotes the Dirac distribution. Since both $T(1, t)$ and $\partial_{x} T(1, t)$ decay rapidly as $t \rightarrow \infty$, all information regarding $T(0, t)$, for $t=0$, is, essentially, contained in a finite interval, say $[0, \tau]$. The 

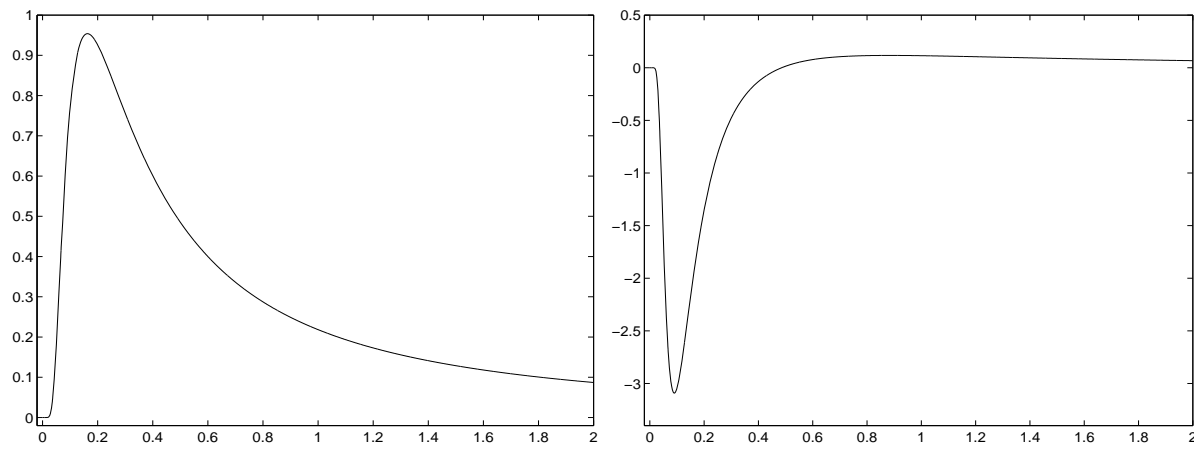

Figure 3: The temperature $T(1, t)$ (left) and heat-flux $\partial_{x} T(1, t)$ (right) corresponding to a situation where the heat equation, $\partial_{x}^{2} T=\partial_{t} T$, is valid for $0<x<5$, and the boundary $x=0$ is subject to a thermal impulse $T(0, t)=\delta(t)$, where $\delta(t)$ is the Dirac distribution.

data $g(t)$ and $h(t)$ for $t>\tau$ contain almost no information regarding $T(0, t)$ for $t<0$. In a practical situation this means that once the measurements for $t<\beta$ have been used, in the sequential algorithm, then the solution $T(0, t)$ will be known, with good accuracy, for $t<\beta-\tau$. Furthermore, future computations using only data, for which $t>\beta$, should not significantly influence the previously obtained results for $t<\beta-\tau$.

The significance of this observation is that it is not really necessary to solve the subproblems for all $t \in \mathbb{R}$. Since the support of the data functions $\left[g_{k}, h_{k}\right]$ is $[k \ell, 1+k \ell]$ sufficient accuracy can be obtained by only solving (9) for a time interval $[k \ell-\gamma, 1+$ $k \ell+\gamma]$, where $\gamma>0$ is large enough, with zero (or periodic) boundary conditions at the ends.

\section{Numerical implementation}

In a series of papers $[2,5,6,15]$ methods for solving the sideways heat equation numerically, where the time derivative $\partial_{t}$ is replaced by a bounded operator, have been investigated. Thus, we discretize the problem in time, and solve it essentially as an initial value problem for a system ordinary differential equations. The resulting numerical method can be considered as a method of lines [8, p. 80].

Let $\left\{t_{k}\right\}_{k=0}^{n-1}$ be an equidistant grid. By discretizing (5) we obtain,

$$
\frac{\partial}{\partial x}\left(\begin{array}{c}
T \\
V
\end{array}\right)=\left(\begin{array}{cc}
0 & \kappa^{-1} I \\
D & 0
\end{array}\right)\left(\begin{array}{l}
T \\
V
\end{array}\right), \quad 0 \leq x \leq 1,
$$

with initial values,

$$
T(1)=G, \quad \text { and }, \quad V(1)=\kappa H,
$$

where $I$ is the unit matrix, $D$ is a discretization of the time derivative, $T$ and $V$ are 
semi-discrete representations of the temperature and the heat-flux respectively, that is,

$$
T(x)=\left(\begin{array}{c}
T\left(x, t_{0}\right) \\
\vdots \\
T\left(x, t_{n-1}\right)
\end{array}\right), \text { and, } V(x)=\left(\begin{array}{c}
V\left(x, t_{0}\right) \\
\vdots \\
V\left(x, t_{n-1}\right)
\end{array}\right),
$$

and the vectors $G$ and $H$ contain samples from $g(t)$ and $h(t)$ on the grid.

The regularized solution (4) can be computed by approximating the time derivative by the matrix,

$$
D=F^{H} \Lambda_{c} F,
$$

where $F$ is the Fourier matrix, and $\Lambda_{c}$ is the diagonal matrix corresponding to differentiation of the trigonometric interpolant, but where the frequency components with $|\xi|>\xi_{c}$ are explicitly set to zero. Thus the diagonal elements of $\Lambda_{c}$ are,

$$
\left(\Lambda_{c}\right)_{k, k}= \begin{cases}i \xi_{k}, & \left|\xi_{k}\right|<\xi_{c} \\ 0, & \left|\xi_{k}\right| \geq \xi_{c}\end{cases}
$$

and the frequencies $\xi_{k}$ depend on the grid $\left\{t_{k}\right\}$. The product of $F$ and a vector can be computed, in only $\mathcal{O}(n \log n)$ operations, using the Fast Fourier transform (FFT).

Note that, by using the FFT algorithm we implicitly assume that the data vectors represents periodic functions. This is not realistic in our application, and this is discussed further in $[2,6]$. Here, the situation is simplified by the fact that in the sequential algorithm we only need to solve problems, where the data have compact support. Suppose that the support of the window function $w(t)$ is the interval $[a, b]$. In order to avoid wrap-around effects we select the time grid $\left\{t_{k}\right\}$ so that $\left[t_{0}, t_{n-1}\right]=[a-\tau, b+\tau]$, where the constant $\tau>0$ is selected large enough so that the difference between a periodic and a non-periodic solution is sufficiently small.

We conclude this section with a brief discussion of the computational costs associated with the proposed sequential procedure. Suppose that the time grid $\left\{t_{k}\right\}$ for the full-domain problem is of size $N_{f}$, and that the width of the window function $w(t)$ corresponds to $N_{w}$ grid points. Thus, instead of solving the full-domain problem once (requiring $\mathcal{O}\left(N_{f} \log \left(N_{f}\right)\right)$ floating point operations) we solve $N_{f} / N_{w}$ different subproblems. Suppose that the grid size for each subproblem is $C_{w} N_{w}$ (for the numerical experiment presented in the next section $C_{w} \approx 3$ ). Then the work required for solving the full-domain problem, using the sequential algorithm, is $\mathcal{O}\left(C_{w} N_{f} \log \left(C_{w} N_{w}\right)\right)$ operations. Thus, the total amount of work is approximately the same for both approaches. Of course, if the full-domain problem is very large then the sequential method has the advantage of dividing the computational work in manageble pieces.

\section{Numerical Experiment}

In this section we present a numerical experiment intended to demonstrate that the proposed method works well. The tests were conducted using MATLAB ${ }^{3}$ (with IEEE dou-

\footnotetext{
${ }^{3}$ The MATLAB programs that were used for creating the graphs displayed in this section are freely available on the Internet address www.math. liu.se/ frber/archive/seqsolv.html
} 


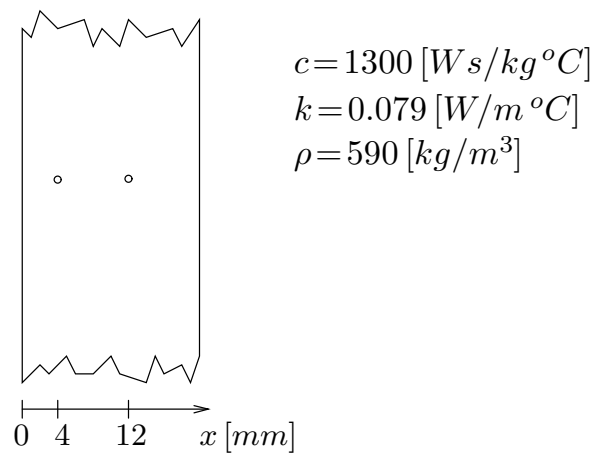

Figure 4: The cross section, in principle, of the particle board. The locations of the thermocouples are marked by circles. The material parameters for the particle board were found in [10, p. 640].

ble precision arithmetic). The space marching was performed using a Runge-KuttaFehlberg method (ode45 in MATLAB) with automatic step size control, where the basic method is of order 4 and the embedded method is of order 5 .

Consider a particle board, on which a thin lacquer coating is to be applied. Since the temperature and heat-flux on and close to the surface influence the drying time and the quality of the lacquer coating it is important to estimate both the temperature and the heat-flux on the surface of the board. This problem was previously studied in $[3,6]$, where a more detailed description of the experimental setup can be found. The test presented here do not involve any actual measured data. For this experiment, the heat equation,

$$
k \frac{\partial^{2} T}{\partial x^{2}}=c \rho \frac{\partial T}{\partial t},
$$

where $k$ is the thermal conductivity, $c$ is the specific heat capacity, and $\rho$ is the density, is valid in an interval $0<x<20 \mathrm{~mm}$. Two thermocouples are located inside the particle board, at distance $x=4 \mathrm{~mm}$ and $x=12 \mathrm{~mm}$ from the surface.

The test problem was created as follows. First we selected the (unknown) boundary temperatures $T(0, t)$ and $T(20, t)$. The corresponding temperature histories, $T(4, t)$ and $T(12, t)$, at the locations of the thermocouples, were then computed using a finite difference scheme. Normally distributed noise, with standard deviation $\sigma=0.2$, were then added in order to simulate measurement errors. Finally, the heat-flux $\partial_{x} T(4, t)$ was computed, by solving a well-posed heat conduction problem, in the region $4<x<$ 12 , with (noisy) boundary data, i.e. $T(4, t)$ and $T(12, t)$, using the Crank-Nicholson scheme [16]. The noisy temperature and heat-flux data, $T(4, t)$ and $\partial_{x} T(4, t)$ are illustrated in Figure 5. The sampling rate is $10 \mathrm{~Hz}$, and the test data were computed for a period of 30 minutes. Thus the length of the data vectors is $18 \cdot 10^{3}$.

In the sequential algorithm we need to solve several sub problems, with compactly supported data, using the numerical procedure described in Section 4. The window was constructed by sampling the function $w(t)$, defined by (7), on a uniform grid of size $2.4 \cdot 10^{3}$. Thus the support of the window cover a period of four minutes. In order to avoid wrap around effects, zeros were added so that the total length of the data vectors 

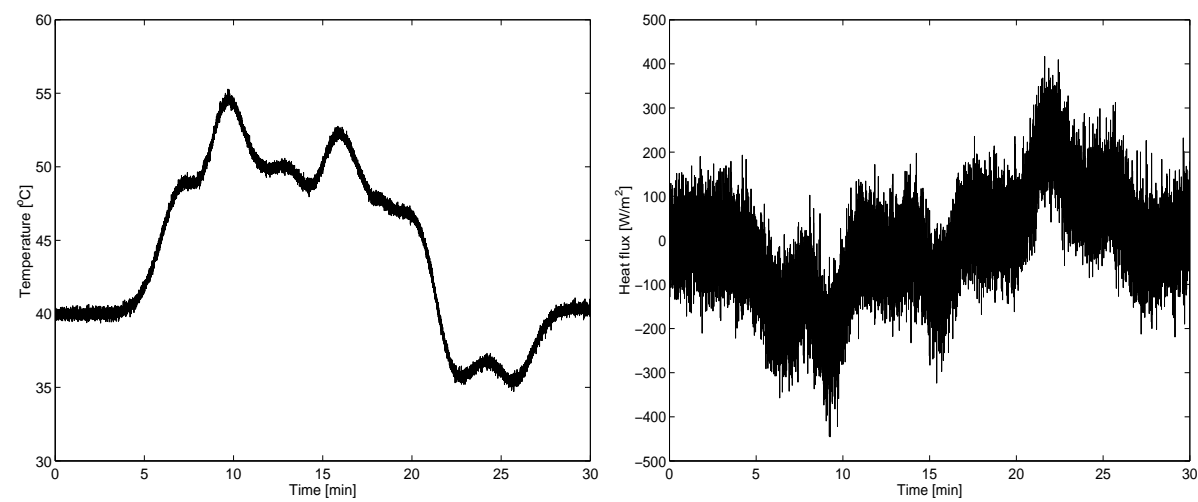

Figure 5: The temperature $T(4, t)$ (left) and the heat-flux $k \partial_{x} T(4, t)$ (right). These, quite noisy, data vectors were used for computing, approximately, the surface temperature $T(0, t)$.

was $7.2 \cdot 10^{3}$. This means that each sub problem is solved for a time period consisting of twelve minutes.

The error estimate, and the rule for selecting the regularization parameter, mentioned in Section 2, was derived for an equation in non-dimensional form, where the location of the thermocouple is $x^{\prime}=1$ and the data are available for the interval $t^{\prime} \in[0,1]$. If the problem under consideration is transformed into non-dimensional form, cf. (11), we find that $\kappa \approx 4.11$ and that the appropriate cut-off level is $\xi_{c}=108$.

In Figure 6 we display the reconstructed surface temperature using between two and three terms in the series (10). Also, the temperature data $g_{3}(t)$ and the solution $T_{3}(0, t)$, for the third sub problem, are displayed. The approximate surface temperatures obtained by using four and five terms in the series (10) are displayed in Figure 7. The solutions $T_{4}(0, t)$ and $T_{5}(0, t)$ are also displayed. More numerical results are presented in Figure 8. In all cases the accuracy of the numerical solution is acceptable.

For this experiment the exact surface temperature, i.e. $T(0, t)$, was selected so that it would be fairly easy to reconstruct, with the exception of one short spike, located at $t=17.8$ minutes. The spike lasts for about 12 seconds, and cannot be reconstructed with any accuracy, as seen in Figure 9. Such fast varying parts of the surface temperature, are almost completely damped out before the signal reaches the recording device. Thus, considering the noise level, the measured data does not contain sufficient information to allow for a good reconstruction.

\section{Concluding remarks}

The proposed numerical method is based on discretizing the problem in the time variable, and thus replacing the time derivative by a matrix, representing differentiation of the trigonometric interpolant. The space discretization is handled by a standard Runge-Kutta code. In our implementation two FFT's are computed at each step of 

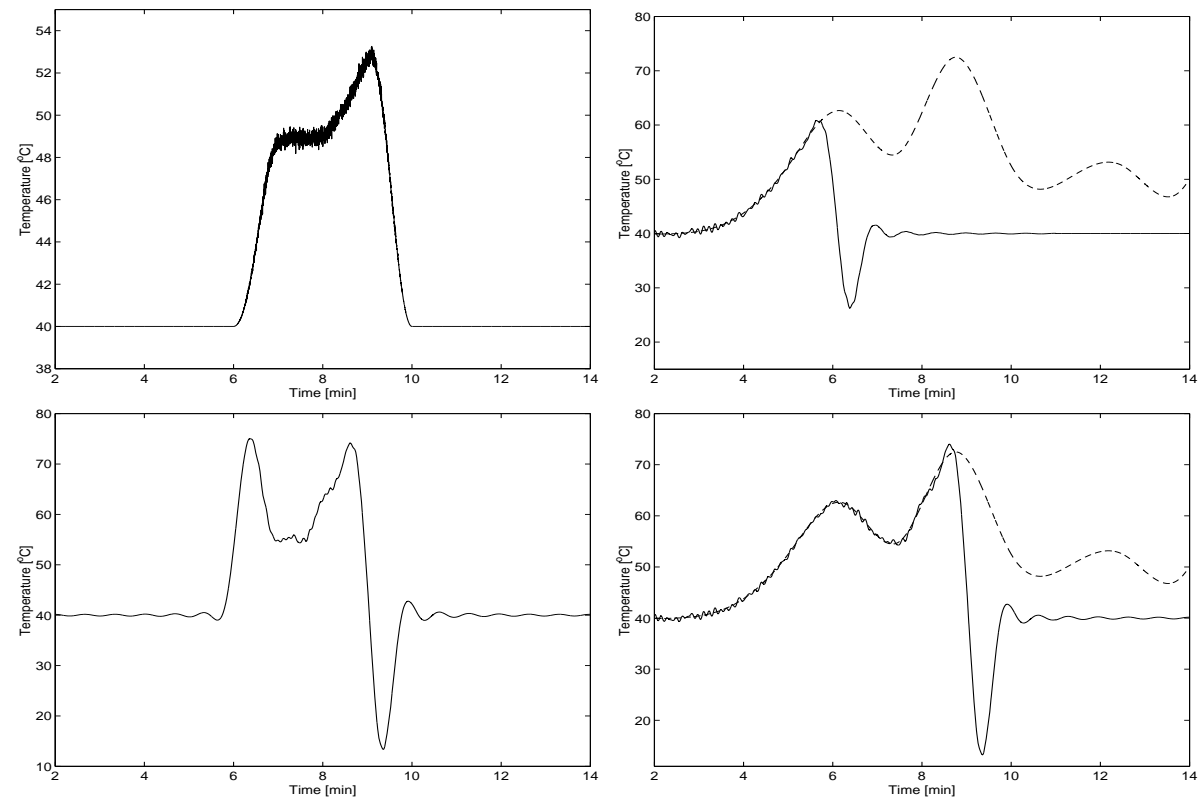

Figure 6: The numerical results after two and three steps of the sequential algorithm. The temperature data $g_{3}(t)$ (upper, left graph) and the solution $T_{3}(0, t)$ (lower, left graph), for the third sub problem are displayed. Also, the results after adding two (upper, right graph) and three terms (bottom, right graph) to the series (10) are displayed. In both these graphs the exact surface temperature is also displayed (dashed curve). Note that, for the last case, data in the interval $t<10 \mathrm{~min}$ have been used, and the computed solution is accurate for $t<8.7 \mathrm{~min}$.

the Runge-Kutta method. The resulting numerical method is easy to program and it is flexible, in the sense that it can easily be adapted for solving more general equations, e.g. (2) and (3).

The main contribution that is made in this paper is the windowing procedure that allows us to solve the problem sequentially in time, using numerical methods which themselves are not sequential. The procedure makes explicit use of the fact the differential equation is linear. Therefore it can also be used for the equation (2), but not for the non-linear equation (3).

The regularization parameter, i.e. the cut off level $\xi_{c}$, was selected using a priori information about the solution, and knowledge of the noise level in the data vectors. Such information may not be available in practice. However, the quality of the numerical solution is not very sensitive to variations of the cut of level, and it is the author's experience that, in practice, it is relatively easy to find an appropriate value for $\xi_{c}$. 

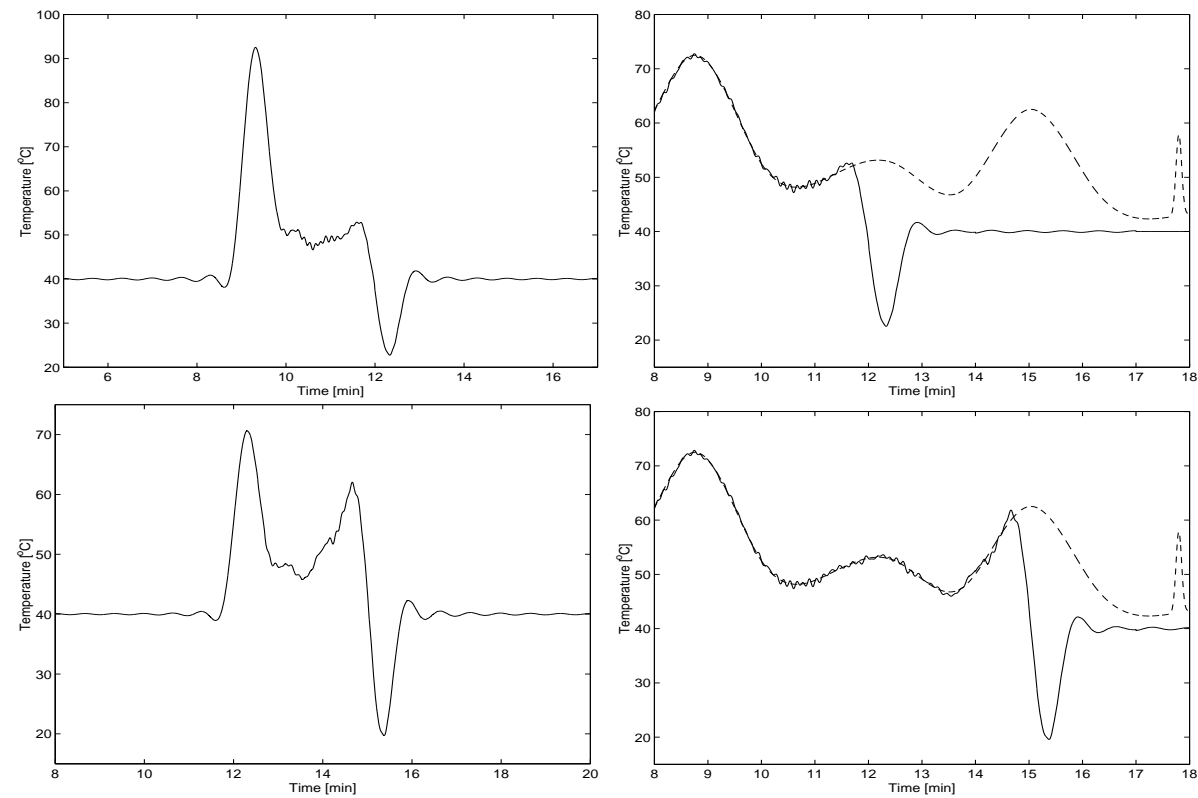

Figure 7: The numerical results after four and five steps of the sequential algorithm. We display the solutions $T_{4}(0, t)$ (upper, left graph) and $T_{5}(0, t)$ (lower, left graph), of the fourth and fifth subproblems respectively. Also, the approximate surface temperature, computed using four (upper, right graph) and five (lower, right graph) terms in the series (10) are presented. In both these graphs the exact surface temperature is also displayed (dashed curve).
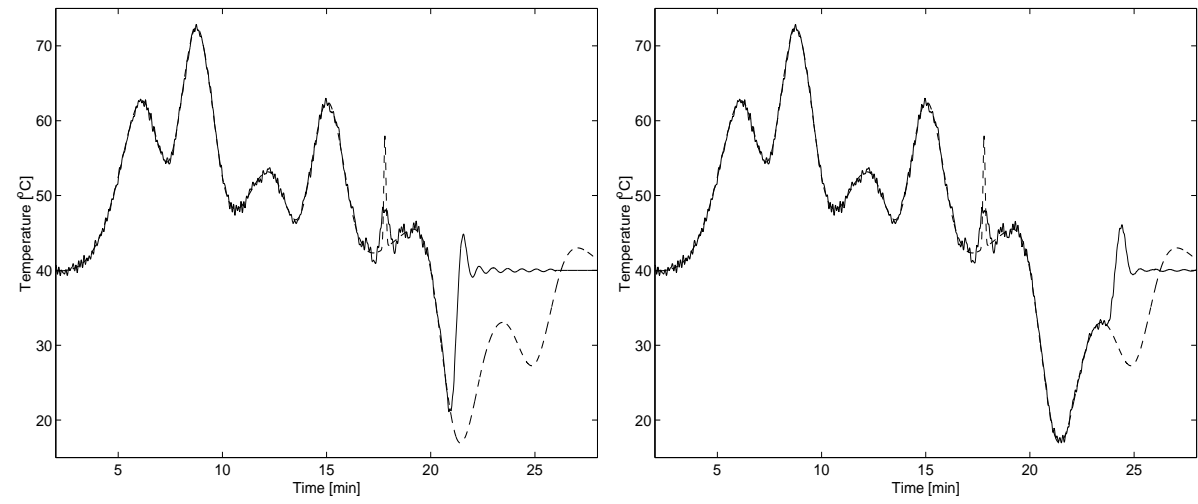

Figure 8: The computed approximations of the surface temperature using seven (left graph, solid curve) and eight (right graph, solid curve) terms in the series (10). Also, the exact surface temperature is displayed (dashed curves). 


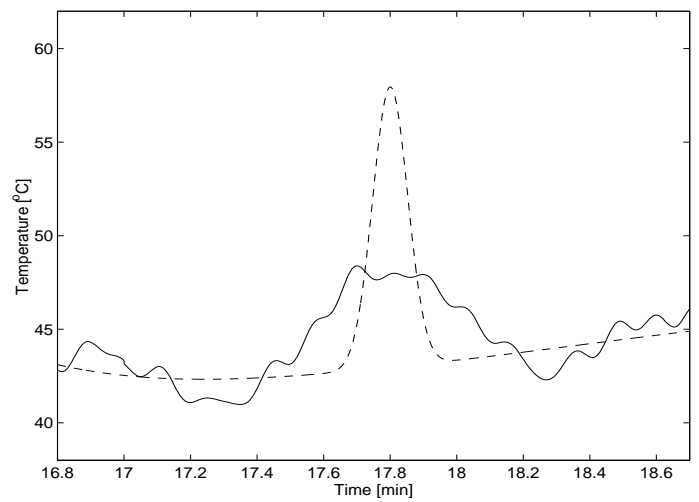

Figure 9: The computed surface temperature (solid curve) in the region close to spike, also the exact temperature (dashed curve) is displayed. Note, that it is not possible to resolve the spike with any reasonable accuracy.

\section{References}

[1] J. V. Beck and B. Blackwell. Inverse problems. In W. J. Minkowycz, E. M. Sparrow, G. E. Scheider, and R. H. Pletcher, editors, Handbook of Numerical Heat Transfer, chapter 19, pages 787-834. J. Wiley and Sons, New York, 1988.

[2] F. Berntsson. A spectral method for solving the sideways heat equation. Inverse Problems, 15:891-906, 1999.

[3] F. Berntsson, L. Eldén, R. Garcia-Padron, and D. Loyd. A comparison of three numerical methods for an inverse heat conduction problem and an industrial application. In R. W. Lewis, editor, Proceedings of the 10th International Conference on Numerical Methods in Thermal Problems, Swansea, 1997.

[4] A. S. Carasso. Determining surface temperatures from interior observations. SIAM J. Appl. Math., 42:558-574, 1982.

[5] L. Eldén. Solving the sideways heat equation by a 'method of lines'. J. Heat Transfer, Trans. ASME, 119:406-412, 1997.

[6] L. Eldén, F. Berntsson, and T. Regińska. Wavelet and Fourier methods for solving the sideways heat equation. SIAM J. Sci. Comput., 21(6):2187-2205, 2000.

[7] H. Engl, M. Hanke, and A. Neubauer. Regularization of Inverse Problems. Kluwer Academic Publishers, Dordrecht, the Netherlands, 1996.

[8] B. Gustafsson, H.-O. Kreiss, and J. Oliger. Time Dependent Problems and Difference Methods. Wiley Interscience, New York, 1995.

[9] Dinh Nho Hào, H.-J. Reinhardt, and A. Schneider. Numerical solution to a sideways parabolic equation. Internat. J. Numer. Methods Engrg., 50(5):1253-1267, 2001. 
[10] J. P. Holman. Heat Transfer, 7th ed. McGraw Hill, 1992.

[11] P. Lamm. Future-sequential regularization methods for ill-posed Volterra equations. Applications to the inverse heat conduction problem. J. Math. Anal. Appl., 195(2):469-494, 1995.

[12] H.A. Levine. Continuous data dependence, regularization, and a three lines theorem for the heat equation with data in a space like direction. Ann. Mat. Pura Appl. (IV), CXXXIV:267-286, 1983.

[13] C. E. Mejía and D. A. Murio. Numerical solution of generalized IHCP by discrete mollification. Computers Math. Appl., 32:33-50, 1996.

[14] D. A. Murio. The Mollification Method and the Numerical Solution of Ill-Posed Problems. J. Wiley \& Sons, New York, 1993.

[15] T. Regińska and L. Eldén. Solving the sideways heat equation by a waveletGalerkin method. Inverse Problems, 13:1093-1106, 1997.

[16] G. D. Smith. Numerical Solution of Partial Differential Equations. Oxford University Press, Oxford, third edition, 1985.

[17] U. Tautenhahn. Optimal stable approximations for the sideways heat equation. $J$. Inv. Ill-Posed Problems, 5:287-307, 1997.

[18] S. Zhan and D. A. Murio. Identification of parameters in one-dimensional IHCP. Comput. Math. Appl., 35(3):1-16, 1998. 